findings are similar to those seen in more common pathologies making it a challenging diagnosis if the entity is not a consideration in the initial evaluation. RCVS presentation and initial imaging findings may mimic a ruptured aneurysm, stroke, migraine and posterior reversible encephalopathy syndrome, among others. Our aim is to describe the entity of RCVS and demonstrate the common and uncommon imaging findings seen on CT, MRI, CT angiography, with emphasis on conventional digital subtraction arteriogram pre and post endovascular treatment.

Approach/methods A multimodality pictorial review of cases from our institution is presented, including non-contrast head CT, CT angiogram, MRI, MR angiogram and conventional digital subtraction arteriogram (DSA). Diagnosis of RCVS was confirmed by imaging demonstration of reversibility of arterial constriction on follow-up imaging or after intra-arterial therapy.

Findings/discussion We present indirect findings on non-invasive imaging, such as transient cerebral edema, convexal subarachnoid hemorrhage, intraparenchymal hemorrhage and cerebral. Direct signs of single or multivessel arterial vasoconstriction are seen on CTA, MRA and confirmed on DSA. Reversibility of the vasoconstriction is demonstrated after intra-arterial administration of calcium channel blocker. Conditions such as diffuse atherosclerotic arterial narrowing, vasculitis, posterior reversible encephalopathy, cerebral edema secondary to venous congestion and vasospasm secondary to aneurysmal subarachnoid hemorrhage were among the pathologies considered prior to a definitive diagnosis of RCVS.

Summary/conclusion Familiarity with the imaging findings of RCVS is of critical importance to direct appropriate and timely clinical management. Atypical subarachnoid hemorrhage, unexplained cerebral edema and hemorrhage, as well as single or multivessel diffuse narrowing with a relatively normal appearing brain should raise suspicion of RCVS. Suspicion can be confirmed by demonstrating the reversibility of vasoconstrictions after intra-arterial administration of calcium channel blocker or at follow-up imaging.

Disclosures K. de Macedo Rodrigues: None. R. Hito: None. D. Takhtani: None. J. Lozano: None. A. Wakhloo: 1; C; NIH, Philips Healthcare, Wyss Institute. 2; C; Codman Neurovascular and Stryker Neurovascular. 4; C; co-founder of InNeuroCo Inc. and major stockholder; stocks in EpiEB and Pulsar Medical.. 6; C; speaker: Harvard Postgraduate Course, Miami Cardiovascular Institute. A. Puri: 1; C; Stryker Neurovascular and Covidien. 2; C; Codman Neurovascular, Stryker Neurovascular and Covidien. 4; C; InNeuroCo Inc. 6; C; speaker: Miami Cardiovascular Institute.

\section{E-044 STENT RETRIEVER THROMBECTOMY AND LOCAL ASPIRATION WITH A NAVIGABLE AND FLEXIBLE SECOND GENERATION SUPPORT CATHETER FOR ACUTE ISCHEMIC STROKE: THE "SOL-ARC" TECHNIQUE}

A Onofrio, A Miller, J Hise, I Thacker, J Haithcock, D Graybeal, K Layton. Radiology, Baylor University Medical Center, Dallas, TX

\subsection{6/neurintsurg-2016-012589.116}

Multiple recent randomized controlled trials have proven the benefit of mechanical thrombectomy using stent retrievers for emergent large vessel occlusion (ELVO). ${ }^{1-5}$ Techniques currently used for endovascular treatment of stroke employ either direct aspiration, stent retriever thrombectomy or a combination of both. When stent retrievers are used, temporary flow arrest with an extracranial balloon guide catheter or assisted local aspiration with a large bore intracranial suction catheter is recommended. This allows for more complete recanalization and prevents embolization to previously uninvolved territories. Recent advancements in large bore intracranial suction catheter technology have made it easier to utilize coaxial stent retriever thrombectomy assisted by local aspiration. At our high-volume comprehensive stroke center, we have found the second generation large bore intracranial Arc support catheter (ev3 Neurovascular, Irvine, CA) to be extremely effective when used in combination with the Solitaire stent retriever (ev3 Neurovascular, Irvine, CA). We have found the Arc support catheter to be much more navigable, less prone to kinking and easier to deliver into the M1 segment (without causing spasm) than the first generation local aspiration catheters. Additionally, the Arc support catheter is less costly than the currently available suction catheters. The Sol-Arc technique begins with placement of a stent retriever device across the embolic occlusion by deployment though a 021 or 027 microcatheter. This microcatheter is placed coaxially through the Arc support catheter which is positioned just proximal to the embolus. After waiting 5 minutes, the stent retriever is pulled inside the Arc support catheter which is simultaneously aspirated. Subsequently, the Arc support catheter is removed while aspirating the guiding sheath in the neck. This technique should allow for faster, safer and more successful stent retriever thrombectomy when used in conjunction with local aspiration.

Disclosures A. Onofrio: None. A. Miller: None. J. Hise: None. I. Thacker: None. J. Haithcock: None. D. Graybeal: None. K. Layton: None.

\section{E-045 PRODUCT COST AND UTILIZATION IN MECHANICAL THROMBECTOMY: A COMPARISON OF TECHNIQUES}

${ }^{1} \mathrm{P}$ Brown, ${ }^{2} \mathrm{M}$ Cobb, ${ }^{1} \mathrm{~T}$ Smith, ${ }^{2} \mathrm{~A}$ Zomorodi, ${ }^{2} \mathrm{~L}$ Gonzalez. ${ }^{1}$ Radiology, Duke University, Durham, NC; ${ }^{2}$ Neurosurgery, Duke University, Durham, NC

\subsection{6/neurintsurg-2016-012589.117}

Introduction/purpose Interventional stroke management has seen a period of explosive growth in the wake of recent trials demonstrating improved outcomes in patients who undergo timely and appropriate procedural management. The two most common interventional techniques involve the use of stent retrieval devices and suction aspiration. While clear clinical benefit to the patient has been seen in the use of these devices, it is not altogether clear the financial implications to the patient and/or his or her insurer for the use of these devices. In a period of heightened sensitivity to healthcare costs, it is reasonable to consider the material cost to the patient and healthcare system for utilization of these new techniques. The goal of our study is to determine the difference, if any, in cost to the healthcare system in performance of interventional thrombectomy by stent retrieval or suction aspiration.

Materials and methods We examined data for interventional stroke management cases performed for M1 segment occlusions at Duke University Medical Center from the period of January 2014 to January 2016. We analyzed the amount billed to the patient for products utilized during these cases. Cases were subdivided into those only utilizing stent retrieval devices (Solitaire $^{\mathrm{TM}}$; Covidien, Dublin, Ireland) and those only 